\title{
Vertebroplasty vs. SHAM for Treating Osteoporotic Vertebral Compression Fractures: A Double Blind RCT (VOPE)
}

\author{
Emil Jesper Hansen*, Ane Simony, Leah Carreon, Rikke Rousing, Hans Tropp and Mikkel Osterheden Andersen \\ Sector of Spine Surgery and Research, Middelfart Hospital, Ostre Hougvej 55, 5500 Middelfart, Denmark
}

${ }^{\star}$ Corresponding author: Emil Jesper Hansen, MD PhD, Center for Spine Surgery and Research, Middelfart Hospital, Ostre Hougvej 55, 5500 Middelfart, Denmark; Phone: +45221367 20; Fax: +4563484281; Email: emiljh@mail.dk

Received: August 28, 2019; Accepted: September 13, 2019; Published: September 20, 2019;

\begin{abstract}
Introduction: Osteoporotic Vertebral Compression Fractures (VCF) affects 20\% of postmenopausal women and can lead to long-term disability.

The effect of Percutaneous Vertebroplasty (PVP) has been debated, since two double-blind RCTs was performed. Our purpose was to investigate the clinical effects of PVP compared with a SHAM procedure in acute osteoporotic VCFs focusing on VAS during activity.

Methods: 52 patients were included in the study, and randomized to either PVP or SHAM. 6 patients were excluded during the study, due to malignancy or need for further surgery. Patients, investigators collecting data, and the statisticians were blinded.

Results: 46 patients were eligible for statistical analysis, 22 patients in the PVP group and 24 patients in the SHAM group. In both groups the VASscores, and HRQL scores improved significantly from baseline values $(\mathrm{p}<0.05)$. There was a statistical significant higher VAS-score in the SHAM-group throughout the trial period ( $\mathrm{p}=0.001$ ), with main contribution from VAS at forward bending.

Conclusion: Our study shows statistical significant higher VAS-score in the SHAM group during the trial period, both groups improved significantly in all clinical parameters. However the limitations of the study and the data at hand do not provide sufficient evidence of the benefits of PVP for treating osteoporotic vertebral compression fractures. Focus in the future of PVP and acute VCFs must be on the 3 months convalescence period and the cost benefit analysis of early mobilization.
\end{abstract}

\section{Introduction}

Osteoporosis is a generalized disease of the bones defined by reduced bone mass. According to the WHO, osteoporosis is defined by bone mass 2.5 Standard Deviations (SD) below peak bone mass. Bone Mineral Density (BMD) can be measured by a DEXA-scan. The $\mathrm{T}$-score indicates if $\mathrm{BMD}$ is above or below peak bone mass. A T-score $<-2.5$ is by definition, osteoporosis. Using this definition every third woman above 50 years old has osteoporosis $[1,2]$.

Osteoporosis is seen in women twice as often than in men. The risk of having an osteoporotic fracture increases with age. Osteoporosis occurs due to age-related loss of bone mass and loss of bone mass caused by other life processes, most important of which is the reduced level of estrogen in postmenopausal women [3].

In many women, there is a general reduction in height caused by compression fractures in vertebral bodies. A vertebral fracture can cause back pain, a kyphotic deformity and reduce pulmonary function when they occur in the thoracic spine [4].

Percutaneous Vertebroplasty (PVP) involves the percutaneous injection of bone cement into fractured vertebrae. PVP is indicated for Vertebral Compression Fractures (VCFs) due to osteoporosis, metastatic disease, multiple myeloma or hemangioma. The method was developed in 1980s in France for the treatment of vertebral hemangiomas and osteolytic vertebral tumors [5]. Indications were later expanded to include osteoporotic VCFs [6]. The method is described safe, with very few complications and can be performed in general anaesthesia or local anaesthesia [6,7].

Worldwide 3 non-blinded RCTs has been performed, where the effect of PVP has been compared with conservative treatment $[10,11,12]$ and 3 RCTs where PVP was compared with a shamprocedure, periostal injection of lidocaine $[8,9,20]$. All of these studies have investigated the effect on patients with osteoporotic VCFs. The non-blinded trial published by Voormolen et al where terminated after 2 weeks as most of the conservative patients crossed over to the PVP group [11].

The other non-blinded trial published by Rousing et al, included patients with acute back pain and VCF. They randomized between PVP and conservative brace treatment [12]. Significant pain relief was noted 4-24 hours postoperatively in PVP group. At 3 and 12 months follow-up there was no significant difference in pain level, physical performance evaluated by sit down testing, between the two treatment arms. The most recent non-blinded RCT published in 2016 involving 107 patients found significant pain relieve in patients with PVP acute osteoporotic VCFs compared with conservative treatment consisting of 2 weeks bed rest, optimal pain medication and prescription of physiotherapy [10]. 
The two RCTs by Buchbinder et al and Kalmes et al $[8,9]$ showed no significant difference in pain relief between PVP and the shamprocedure, and not a substantial relief of pain in general. In the study by Kalmes [9] the patients included had a history of back pain up to 52 weeks, and a total of 131 patients included. Amount of PMMA cement injected was not recorded. In the study by Buchbinder [8] including 78 patients the patients were included both with and without edema present on the MRI scan and a VAS score at inclusion from 30-100.

The primary purpose of this study was to compare the Visual Analog Back Pain scores (VAS) [13] at rest and with during mobilization weekly during the first 12 weeks in a double-blind placebo-controlled RCT of PVP vs. SHAM for acute osteoporotic VCFs. Secondary outcome measures of interest were improvements in the EuroQOL-5D (EQ5D) [14] and the Short Form-36 Physical Composite Summary Scores (SF-36 PCS) [15] compared to baseline one year after the procedure.

\section{Methods}

\section{Study Design}

A double-blinded placebo-controlled RCT to determine the efficacy of PVP in patients with acute osteoporotic VCFs.

The study was approved by the regional Ethics Committee and was registered on clinicaltrials.gov (NCT\#01537770). Trial protocol as approved by the ethics committee is displayed at the above mentioned NCT\#. Partially funded by the Danish Rheumatism association.

\section{Inclusion Criteria}

Osteoporotic VCF from T5-L5, >70 in VAS at Inclusion, $</=$ 8 weeks of back pain and a Magnetic Resonance Imaging Short Tau Inversion Recovery(MRI-STIR) sequence showing edema using a Philips Achieva 1.5 Tesla scanner, (Andover, MA).

\section{Exclusion Criteria}

Patients with a history of malignancy, age below 50 years, known allergy towards PVP components, dementia as determined on the MMSE [16], osteoporotic fractures of the long bones and those unable to consent were excluded.

Level of evidence: 1

Randomization was a block randomization design using 80 sealed envelopes (blocks of 20). Procedures were performed under local anaesthesia using the V-Max Mixing and Delivery System (DePuy Acromed). Subjects were placed prone on a Jackson table and Lidocaine was used to anesthetize the entry points. The 11-gauge needles were then inserted into the fractured vertebral body via the pedicles under fluoroscopic guidance and a biopsy specimen was taken. In all cases, bone cement was mixed to create the odor similar to a PVP-procedure. $2 \mathrm{ml}$ of Lidocaine $(10 \mathrm{mg} / \mathrm{ml})$ was injected in the SHAM group. 2-4 $\mathrm{ml}$ of bone cement was injected in the PVP group.

VAS at rest and during forward bending was collected at enrollment, 6 hours postoperatively, weekly for the first 3 months and at 1 year.. A Danish version of SF-36 [15], EQ5D [14], data on pain medication use and standing full-length spine radiographs were collected at enrollment and at 3 and 12 months. Blood samples were also drawn and analysed to exclude infections and malignancies.

The primary investigator performed all screening procedures and follow-up examinations, and was blinded to the subject's assigned treatment arm throughout the study period.

\section{Statistical Analysis}

\section{Power analysis}

Power calculation was performed to find a difference in VAS of $20(0-100)$ with SD of 0.20. A difference of 20 was chosen, as this was past the Minimal Clinical Important Difference (MCID) reported in other studies $[17,18]$. This resulted in a power calculation suggesting a minimum of 23 patients needed in each group. Our aim prior to enrolment was 40 in each group.

The statistical analyses were conducted in SAS version 9,4 (SAS institute, Carry, NC). Repeated measures ANOVA was used to determine differences in VAS between PVP and Sham group. Unpaired student's t-test was used to compare continuous variables and Fisher's exact test was used to compare categorical variables between groups.

Least-square Means and standard errors of pain scores from a numerical self-reported VAS on a scale of 0 to 100 were calculated from measures collected in two different positions - standing and bending forward - at pre-op, at 6 hours post-treatment (rest only), at week 1-12, and at one year post-treatment. A paired t-test or Wilcoxon signed rank test was used to test for differences between baseline and each subsequent time period. Multiple comparisons were adjusted using Tukey's test.

Trends over time, by treatment group and by position are compared using a repeated measures mixed effects model, adjusted for timing past baseline, position (at rest or bending) and type of treatment administered(PVP or SHAM). An independent and blinded statistician performed the statistical analyses.

\section{Results}

A total of 342 potential subjects were referred to our outpatient clinic between 2011-2014 and were screened in order to find eligible patients to include in this study. All patients included signed an informed consent form to participate in a clinical trial, and that the data could be published in a blinded format. The patients were informed of the option of dropping out at any time without any reason needed.

The reasons for not enrolling 290 of the screened patients in the study were mainly that the time from symptom onset was exceeding the 8 week period, at the time they were referred to our clinic, and patients unwillingness to participate in a clinical trial. In total 52 patients were included. During the trial period 2 patients were excluded postoperatively due to malignant biopsies. 4 patients were excluded due to the need for further spine surgery. (Figure 1) No complications including cement leakage or infections occurred perioperative or during the postoperative period. 46 subjects were included in the final analyses; 24 in the Sham procedure and 22 in 
the PVP treatment. There were no differences in patient demographics and Bone Mineral Density t-scores between the two groups.

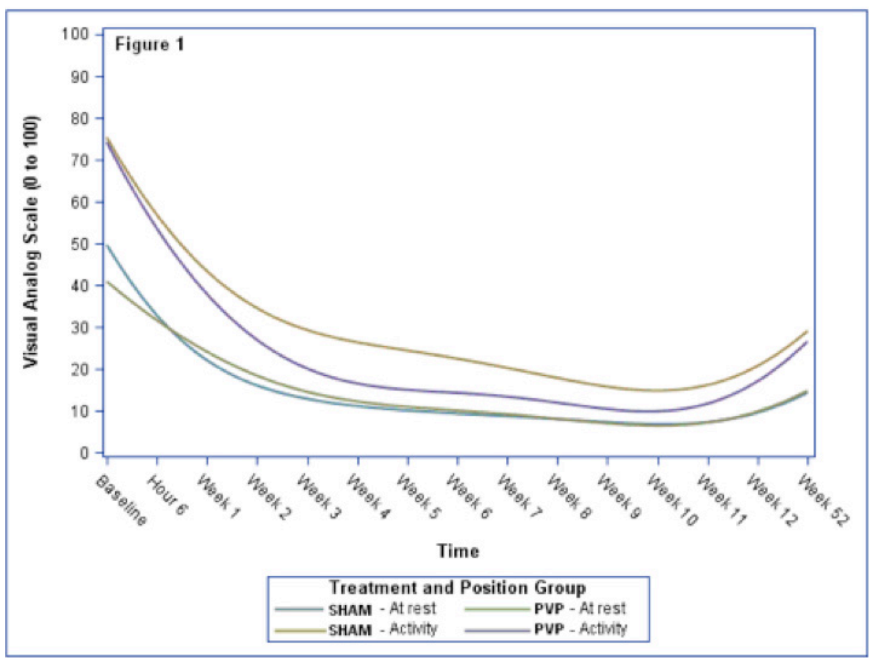

Figure 1.

Table 1. Patient Demographics.

\begin{tabular}{|l|c|c|c|}
\hline \multicolumn{1}{|c|}{ Table 1 } & SHAM & PVP & p-value \\
\hline Age (years) & $69,33(53-84)$ & $70,59(54-90)$ & 0,309 \\
\hline Sex M/F & $2 / 22$ & $4 / 18$ & 0,322 \\
\hline BMD T-score & $-2,2(0,24)$ & $-2,7(0,25)$ & 0,875 \\
\hline No. Levels treated & 28 & 27 & 0,932 \\
\hline New VCFs & $5(21 \%)$ & $4(19 \%)$ & 0,688 \\
\hline Vertebral Levels & Th7-L5 & Th6-L5 & \\
\hline
\end{tabular}

VAS and SE for each time period and position are shown in Table 2: VAS scores 12 months follow-up.

Table 2. Means and Standard Errors of VAS by treatment and Group and position.

\begin{tabular}{|l|c|c|}
\hline \multicolumn{1}{|c|}{ Table 2 } & SHAM & PVP \\
\hline Position & Mean(SE) & Means (SE) \\
\hline Resting & & $40,55(4,55)$ \\
\hline Baseline & $53,04(4,35)$ & $32,76(5,07)$ \\
\hline Hour 6 & $26,45(4,82)$ & $24,52(4,65)$ \\
\hline Week 1 & $21,13(4,35)$ & $17,52(4,65)$ \\
\hline Week 2 & $19,08(4,35)$ & $13,57(4,65)$ \\
\hline Week 3 & $13,87(4,45)$ & $12,62(4,65)$ \\
\hline Week 4 & $10,42(4,35)$ & $11,81(4,65)$ \\
\hline Week 5 & $10,17(4,45)$ & $9,00(4,55)$ \\
\hline Week 6 & $8,83(4,35)$ & $10,52(4,65)$ \\
\hline Week 7 & $8,29(4,35)$ & $8,00(4,65)$ \\
\hline Week 8 & $8,29(4,35)$ & $6,82(4,55)$ \\
\hline Week 9 & $8,04(4,35)$ & \\
\hline
\end{tabular}

\begin{tabular}{|l|c|c|}
\hline \multicolumn{1}{|c|}{ Table 2 } & SHAM & PVP \\
\hline Week 10 & $7,57(4,35)$ & $6,40(4,77)$ \\
\hline Week 11 & $7,21(4,35)$ & $8,64(4,55)$ \\
\hline Week 12 & $6,88(4,35)$ & $16,06(5,03)$ \\
\hline Week 52 & $16,04(4,35)$ & \\
\hline Forward bending & & $74,68(4,55)$ \\
\hline Baseline & $76,08(4,35)$ & $26,80(4,77)$ \\
\hline Week 1 & $41,83(4,45)$ & $28,52(4,65)$ \\
\hline Week 2 & $34,83(4,45)$ & $17,81(4,65)$ \\
\hline Week 3 & $28,83(4,45)$ & $17,33(4,65)$ \\
\hline Week 4 & $26,27(4,55)$ & $14,33(4,65)$ \\
\hline Week 5 & $27,14(4,55)$ & $15,27(4,55)$ \\
\hline Week 6 & $21,09(4,45)$ & $13,62(4,65)$ \\
\hline Week 7 & $19,26(4,45)$ & $10,00(4,55)$ \\
\hline Week 8 & $19,77(4,55)$ & $(4,77)$ \\
\hline Week 9 & $15,87(4,45)$ & $(5,16)$ \\
\hline Week 10 & $30,67(4,65)$ & \\
\hline Week 11 & & \\
\hline Week 12 & & \\
\hline Week 52 & & \\
\hline VAS; Visual Analog Scale & & \\
\hline to 100 & & \\
\hline
\end{tabular}

Penalized b-spline curves are shown in Figure 1 by treatment and position. The at-rest position had the lowest VAS regardless of treatment or time from baseline. Study participants in the PVP group had a faster drop in their bending VAS compared to the SHAM group.

There was a difference in treatment groups with the SHAM group having higher overall VAS $(\mathrm{p}=0.011)$. The VAS changes over time with highest levels experienced at baseline through week 3 and increasing moderately during follow-up $(\mathrm{p}<0.0001)$. Forward bending resulted in elevated VAS compared to the at-rest position $(\mathrm{p}<0.0001)$.

We were unable to detect a statistically significant difference between the VAS of the treatment groups at any measured time period within the same position. Before multiple comparison adjustment, there is some suggestion that the baseline VAS in the at-rest position differ by treatment $(\mathrm{p}=0.0476)$.

While there was a significant difference from baseline through week 6 in the SHAM group and at baseline for the PVP group, only the PVP group difference remained significant once adjusted for multiple comparisons(adj-p=0.0002).

No statistical differences were found in SF-36 and EQ-5D scores between the two groups at 3 and 12 months follow-up in any of the parameters analysed. 
Table 3. Health-related Questionnaires.

\begin{tabular}{|l|c|c|c|}
\hline \multicolumn{1}{|c|}{ Table 3 } & SHAM & PVP & p-value \\
\hline SF36 PCS & & & \\
\hline Baseline & $25,53(4,64)$ & $25,12(6,86)$ & 0,406 \\
\hline 3 months & $33,93(10,56)$ & $31,44(10,03)$ & 0,219 \\
\hline 12 months & $35,15(11,92)$ & $31,90(9,19)$ & 0,16 \\
\hline SF36 MCS & & & 0,255 \\
\hline Baseline & $44,29(13,10)$ & $42,00(9,75)$ & 0,318 \\
\hline 3 months & $51,4(10,98)$ & $49,7(12,02)$ & 0,063 \\
\hline 12 months & $53,60(10,29)$ & $48,60(10,75)$ & 0,343 \\
\hline EQ5D & & & 0,34 \\
\hline Baseline & 0,49 & $0,68(0,23)$ & 0,232 \\
\hline 3 months & $0,74(0,22)$ & $0,67(0,27)$ & \\
\hline 12 months & & & \\
\hline
\end{tabular}

At 0-12 weeks and at 12 month follow up there were a similar amount and frequency of opiods in the two groups.

\section{Discussion}

The debate whether the evidence for PVP in acute osteoporotic VCFs is sufficient enough to recommend it as a standard procedure is ongoing. We have focused on patients both at rest and during mobilization, and with a specific focus on the convalescence period the first 3 months after treatment; the latter is in contrary to the other studies on this subject. Thus a direct comparison between our results and the previous SHAM studies by Kalmes and Buchbinder, is difficult, due to the different study designs, the time of pain evaluation during the study, and the evaluation of pain at rest/ during mobilization. There are limitations to our study, the most important ones being the sample size. We did not succeed in our primary aim to include 40 patients in each group, and that weakens the study. Comparing with other studies of this patient group an intervention type, similar difficulties with sample-size and inclusion have been reported. We had a inclusion rate of about $16 \%$ (52/342), the Buchbinder et al. RCT had a $17 \%$ inclusion rate $(52 / 500)$ and the Kalmes et al. RCT had a $13 \%$ rate $(72 / 450)$.

We found in our study a significant higher VAS in the SHAM group throughout the follow up period $(\mathrm{p}=0.001)$ when applying ANOVA statistical model on our data. A study of the minimal clinically important difference in patient with acute pain was $9 \mathrm{~mm}$ (6-13; 95\% CI) in VAS [18]. Other studies have shown a MCID on 13$30 \mathrm{~mm}$, primarily in patients with chronic pain $[17,19]$. The maximum difference measured in our study is at week 3-5 in activity with differences of 9-13. The main contribution to the difference in VAS score were during mobilization and favours PVP treatment specifically in the first 1-12 weeks after treatment. By asking the patients of their back pain in the forward bending position, we resembled a patient moving from lying/sitting position to standing position, with axial load on the fractured vertebrae. A double blinded RCT published in 2016 by Clark et al. found PVP being a superior pain relieving agent compared with a sham procedure, which supports the trend found in our trial. However their sham treatment did not involve periostal infiltration and biopsies from the affected vertebral bodies [20], and their outcome measures regarding pain observation differed from our study and the studies by Buchbinder and Kalmes.

When comparing with the RCTs on PVP vs. SHAM by Buchbinder et al. and Kalmes et al. our findings are comparable when reviewing the Health related Quality of life questionnaires with no difference between the two groups. Our results however contradict in the VAS scores. The findings by Kalmes et al. who found no difference at any time point between the SHAM and PVP group within the first month. Similar the findings by Buchbinder et al. we are not significant between the groups within the first 6 months after surgery. There are several reasons for the difference in findings between the studies. In our opinion the main focus on the back pain in activity and the study design (differences described in the introduction section) is responsible for these differences in findings. It is remarkable that this study with fewer participants were able to detect a statistical significant difference.

\section{Conclusion}

This study set out to investigate whether PVP procedure has its relevance, in treating osteoporotic VCFs, compared with a SHAM procedure. Our study shows statistically significant difference in back pain primarily in forward bending causing significantly more pain in the SHAM group. In acute VCFs it is as well clinically relevant in the early convalescence period. Also, pain decreases over time, regardless of position or treatment and remains decreased from baseline. As suspected, there is no difference when the patients are at rest, with no axial force applied to the fractured vertebrae. With the limitations of this study and the data provided we cannot conclude if and how PVP has its place in treating osteoporotic VCFs. Out study has shown a trend towards a pain-relieving effect when patients are mobilised and applying axial force on the fractured vertebrae, and further studies with this focus and on the cost benefit of early mobilization are needed.

\section{Funding}

The study received funding from The Danish Rheumatism Association.

\section{Trial Registration}

The study was registered at clinical trials.org and approved by the National Ethics committee. 


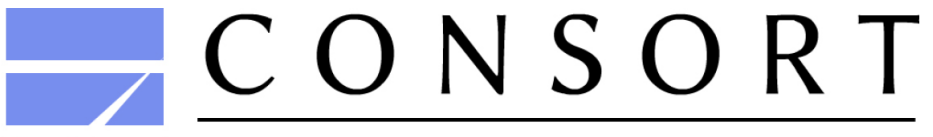

TRANSPARENT REPORTING of TRIALS

\section{CONSORT Flow Diagram}

\section{Enrollment}

Assessed for eligibility $(n=342)$

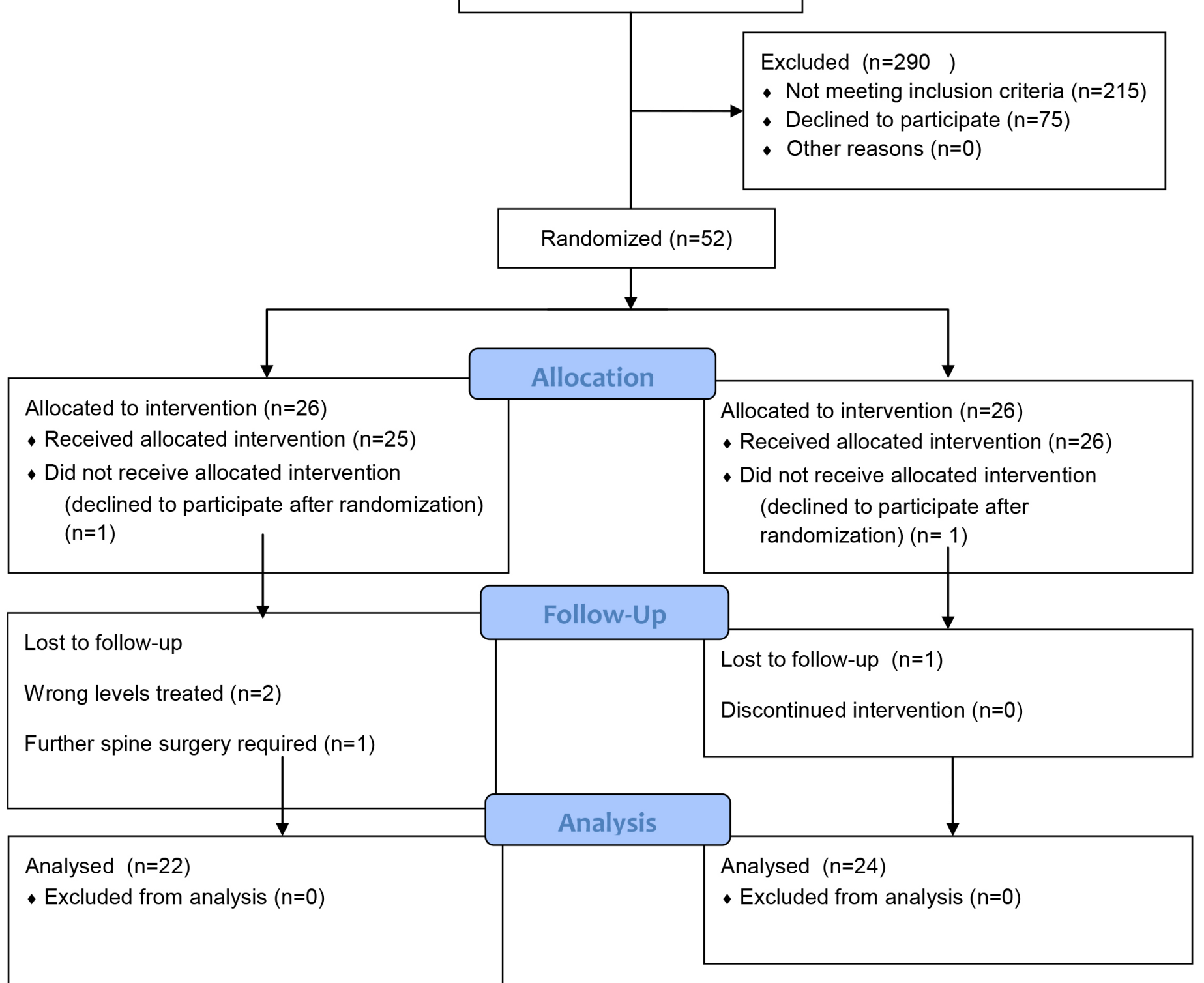




\section{References}

1. Felsenberg D, Silman AJ, Lunt M et al. (2002) Incidence of vertebral fracture in Europe: results from the European Prospective Osteoporosis Study (EPOS). $J$ Bone Mineral Res 17: 716-24.

2. Curtis EM, Moon RJ, Harvey NC et al. (2017) The impact of fragility fracture and approaches to osteoporosis risk assessment worldwide. Bone 22: S8756-3282, 30024-8.

3. Silverman SL, Minshall ME, Shen W et al. (2001) The relationship of health-related quality of life to prevalent and incident vertebral fractures in postmenopausal women with osteoporosis: results from the Multiple Outcomes of Raloxifene Evaluation Study. Arthritis Rheum 44: 2611-2619

4. Suzuki N, Ogikubo O, Hansson T (2008) The course of the acute vertebral body fragility fracture: its effect on pain, disability and quality of life during 12 months. Eur Spine J 17: 1380-1390

5. Galibert P, Deramond H, Rosat $P$ et al. (1987) Preliminary note on the treatment of vertebral angioma by percutaneous acrylic vertebroplasty. Neurochirurgie 33: $166-8$.

6. McGraw JK, Lippert JA, Minkus KD et al. (2002) Prospective Evaluation of Pain Relief in 100 Patients Undergoing Percutaneous Vertebroplasty: Results and Follow-up. Journal of Vascular and Interventional Radiology 13: 883-6.

7. Lee BJ, Lee SR, Yoo TY. Paraplegia as a complication of percutaneous vertebroplasty with polymethylmethacrylate: a case report. Spine 2002;27:E419-E422.

8. Buchbinder R, Osborne RH, Ebeling PR et al.( 2009) A Randomized Trial of Vertebroplasty for Painful Osteoporotic Vertebral Fractures. $N$ Engl J Med 361: $557-68$.

9. Kallmes DF, Comstock BA, Heagerty PJ et al. (2009) A Randomized Trial of Vertebroplasty for Osteoporotic Spinal Fractures. N Engl J Med 361: 569-79.

10. Yang EZ, Xu JG, Huang GZ et al. (2016) Percutaneous Vertebroplasty Versus Conservative Treatment in Aged Patients With Acute Osteoporotic Vertebra Compression Fractures: A Prospective Randomized Controlled Clinical Study. Spine 41: 653-60.

11. Voormolen MH, Mali WP, Lohle PN et al. (2007) Percutaneus vertebroplasty compared with optimal pain medication treatment: short-term clinical outcome of patients with subacute or chronic painful osteoporotic vertebral compression fractures. The VERTOS study. AJNR Am J Neuroradiol 28: 555-60.

12. Rousing R, Hansen KL, Andersen MO et al. (2010) Twelve-Month Follow-up in Forty-Nine Patients With Acute/Semiacute Osteoporotic Vertebral Fractures Treated Conservatively or With Percutaneous Vertebroplasty: A Clinical Randomized Study. Spine 35: 478-82.

13. Jensen MP, Karoly P, Braver S (1986) The measurement of clinical pain intensity: a comparison of six methods. Pain 27: 117-26.

14. Sorensen J, Davidsen M, Gudex C et al. (2009) Danish EQ-5D population norms. Scand J Public Health 37: 67-74. See comment in PubMed Commons below

15. Bjorner JB, Thunedborg K, Kristensen JS et al. (1998) The Danish SF-36 Health Survey: Translation and Preliminary Validity Studies. Journal of Clinical Epidemiology 51: 991-999.

16. Folstein MF, Folstein SE, McHugh PR (1975) Mini Mental State. A practical method for grading the cognitive state of patients for the clinician. J Psychiatri Res 12: 189-198.

17. Kelly AM (1998) Does the clinically significant difference in visual analog scale pain scores vary with gender, age, or cause of pain? Acad Emerg Med 5: 1086-90.

18. Lee JS, Hobden E, Stiell IG (2003) Clinically important change in the visual analog scale after adequate pain control. Acad Emerg Med 10: 1128-30.

19. Hägg O, Fritzell P, Nordwall A (2003) Swedish Lumbar Spine Study Group. The clinical importance of changes in outcome scores after treatment for chronic low back pain. Eur Spine J 12: 12-20.

20. Clark W, Bird P, Gonski P et al (2016) Safety and efficacy of vertebroplasty for acute painful osteoporotic fractures (VAPOUR): a multicentre, randomised, doubleblind, placebo-controlled trial. Lancet 388: 1408-1416.

\section{Citation:}

Hansen EJ, Simony A, Carreon L, Rousing R, Tropp H, Andersen MO (2019) Vertebroplasty vs. SHAM for Treating Osteoporotic Vertebral Compression Fractures: A Double Blind RCT. Integr J Orthop Traumatol Volume 2 (4): 1-6. 